\title{
On self-similar solutions of time and space fractional sub-diffusion equations
}

\author{
Fatma Al-Musalhi ${ }^{a *}$ and Erkinjon Karimov ${ }^{b}{ }^{c}$ \\ ${ }^{a}$ Center for Preparatory Studies, Sultan Qaboos University, Oman \\ ${ }^{b}$ V.I. Romanovskiy Institute of Mathematics, Uzbekistan \\ ${ }^{c}$ Ferghana State University, Uzbekistan \\ fatma@squ.edu.om,erkinjon.karimov@mathinst.uz
}

\section{ARTICLE INFO}

Article History:

Received 21 December 2020

Accepted 22 September 2021

Available 1 November 2021

Keywords:

Self-similar solution

Erdélyi-Kober fractional derivative

Hilfer derivatives

Hyper-Bessel operator

Successive iteration method

\section{ABSTRACT}

In this paper, we have considered two different sub-diffusion equations involving Hilfer, hyper-Bessel and Erdélyi-Kober fractional derivatives. Using a special transformation, we equivalently reduce the considered boundary value problems for fractional partial differential equation to the corresponding problems for ordinary differential equation. An essential role is played by certain properties of Erdélyi-Kober integral and differential operators. We have applied also successive iteration method to obtain self-similar solutions in an explicit form. The obtained self-similar solutions are represented by generalized Wright type function. We have to note that the usage of imposed conditions is important to present self-similar solutions via given data.

$(\mathrm{cc}) \mathrm{EY}$

AMS Classification 2010:

26A33; 33E20; 33E30; 35C06; 45J05

\section{Introduction}

Fractional calculus became one of the intensively developing theories in modern mathematics due to its wide range of applications in real life processes and also its generalized nature [1]. In particular, fractional derivative operators allow the description of memory and hereditary properties and are useful for modeling dynamic. Recently, several fractional operators have been developed to analyze the systems and models such as Caputo-Fabrizio, Hilfer, hyper-Bessel, ErdélyiKober fractional derivatives and many others. For instant, in recent papers [2,3], fractional differential equations are used for modeling applications in blood alcohol and fish farm models and in [4] fractional partial differential equation is used for Frankl-Type Problem.

Fractional order partial differential equations (FPDE) is one of the key objects in mathematical modeling of many diffusion-wave processes
5]. Different kind of direct and inverse problems for such equations were studied using different approaches, such as, integral transformations (Laplace, Fourier, Mellin), Green function method, method of separation of variables and etc. For PDEs, in general, one can determine special type of solutions, which are invariant under some subgroup of the full symmetry group of system. These "group-invariant" solutions are found by solving a reduced system of equations having fewer independent variables than the original system [6]. Such solutions named as self-similar solutions which play an important role in understanding of fundamental processes in mathematics and mechanics, we refer readers to [7] for application in problems of imploding shock waves and to 8] for filtration-slow groundwater motion in porous media.

The self-similarity of the solutions of partial differential equations has allowed their reduction to

*Corresponding Author 
ordinary differential equations, which often simplifies the investigation. They have also served as standards in evaluating approximate methods for solving more complicated problems [8]. Moreover, they often describe the intermediate asymptotics behavior of solutions of wider classes of problems, for more details see [8].

The idea of self-similarity of solutions and Lie group analysis have been extended to fractional differential equations. For instant, in [6] and [9], the Lie group analysis of the equation

$$
\frac{\partial^{\alpha} u}{\partial t^{\alpha}}=d \frac{\partial^{\beta} u}{\partial x^{\beta}}, x>0, t>0, d>0, \alpha, \beta \geq 0
$$

has been discussed by Buckwar, Luchko and Gorenflo. Namely, the scale-invariant solutions were found by solving an ordinary differential equation of fractional order with a new independent variable $\eta=x t^{-\frac{\alpha}{\beta}}$. The general solution for this equation is obtained in terms of the generalized Wright function.

Furthermore, the existence and uniqueness of the space-fractional PDE with Caputo fractional derivative

$$
\frac{\partial u}{\partial t}=\frac{\partial^{\alpha} u}{\partial x^{\alpha}}, 1<\alpha \leq 2
$$

was discussed, under the self-similar form

$$
u(x, t)=t^{\beta} f\left(\frac{x}{t^{1 / \alpha}}\right),(x, t) \in[0, X] \times\left[t_{0}, \infty\right),
$$

where $X, t_{0}>0, \beta \in \mathbb{R}[10]$.

In [6], an admitted group dilations is found for the linear wave-diffusion equation of fractional order and these transformations are used for the construction of self-similar solutions. In [11, the methods of Lie continuous groups for symmetry analysis of FDEs were adapted and prolongation formula for fractional derivatives was proposed. Then, in [12], this formula is used for finding the exact solutions for nonlinear sub-diffusion equations with the Riemann-Liouville and Caputo fractional derivatives.

In [13], the similarity solution of the fractional diffusion equation

$$
\frac{\partial^{\gamma} p(r, t)}{\partial t^{\gamma}}=\frac{1}{r^{d_{s}-1}} \frac{\partial}{\partial r}\left(r^{d_{s}-1} \frac{\partial p}{\partial 2 r}\right), r>, t>0,
$$

$\left(\gamma=\frac{2}{d_{w}}, d_{s}=\frac{2 d_{f}}{d_{w}}\right.$ is the spectral dimension of the fractal) was considered and through the invariants of the group of scaling transformations, authors derived the integro-ordinary differential equation for the similarity variable.

In [14, fractional nonlinear space-time wavediffusion equation was considered and solved by the similarity method using fractional derivatives in the Caputo, Riesz-Feller, and Riesz senses. Some particular cases are presented and the corresponding solutions are shown by means of $2-\mathrm{D}$ and 3-D plots.

The following time-fractional cylindrical KdV equation with Riemann-Liouville fractional derivative

$$
\frac{\partial^{\alpha} u}{\partial t^{\alpha}}+\frac{u}{2 t^{\alpha}}+6 u \frac{\partial u}{\partial x}+\frac{\partial^{3} u}{\partial x^{3}}=0, \alpha \in(0,1)
$$

was reduced to the nonlinear fractional ordinary differential equation with Erdélyi-Kober fractional differential operator, using similarity transformation $u(x, t)=t^{-\frac{2 \alpha}{3}} f(z)$ along with the similarity variable $z=x t^{-\frac{\alpha}{3}}$ [15].

There are other approaches, were authors have found self-similar solution by reducing considered PDEs to the hypergeometric equations. For example, Hasanov and Ruzhansky have found selfsimilar solutions for degenerate PDEs of the second, third and fourth orders using special method (see for details [16]). Precisely, they considered the following fourth order degenerate PDE:

$$
x^{n} u_{t}-t^{k} u_{x x x x}=0, \quad n, k=\text { const }>0 .
$$

They are looking for a solution of this equation as

$$
u(x, t)=P(t) \omega(\sigma),
$$

where

$$
P=\left(\frac{1}{k+1} t^{k+1}\right)^{-1}, \sigma=-\frac{k+1}{(n+4)^{4} t^{k+1}} x^{n+4} .
$$

Then they have got the equation with respect to $\omega$ :

$$
\begin{aligned}
& x^{3} \omega_{x x x x}+\left(3+c_{1}+c_{2}+c_{3}\right) x^{2} \omega_{x x x} \\
& +\left(1+c_{1}+c_{2}+c_{3}+c_{1} c_{2}+c_{1} c_{3}+c_{2} c_{3}\right) x \omega_{x x}+ \\
& \left(c_{1} c_{2} c_{3}-x\right) \omega_{x}-a \omega=0
\end{aligned}
$$

which has special solutions represented with hypergeometric functions ${ }_{p} F_{q}$.

The main motivation of the present research is the consideration of combinations of special fractional derivatives such as hyper-Bessel, Erdélyi-Kober 
(due to singularity) and Hilfer (due to generalized character). The obtained self-similar solutions will allow specialists in applied mathematics, who may deal with such FPDEs to study in details, since an explicit form of solutions are available. Moreover,the offered approach can be developed to conduct further investigations for more general FPDE with aforementioned fractional derivatives and also will contribute in studying the symmetry group analysis of FPDEs with these derivatives. In the present paper, we consider two problems, namely, fractional differential equation involving time and space Hilfer derivatives

$D_{0 t}^{\alpha, \delta} u(t, x)=D_{0 x}^{\beta, \delta} u(t, x), \quad 0<\alpha \leq 1,1<\beta \leq 2$,

and fractional differential equation involving hyper-Bessel operator in time and Erdélyi-Kober fractional derivative in space variable

$$
\left(t^{\theta} \frac{\partial}{\partial t}\right)^{\alpha} u(t, x)=x^{-\beta \rho} \frac{\partial^{\beta}}{\partial x^{\beta}} u(t, x),
$$

where $1<\beta \leq 2,0<\alpha \leq 1$.

The key result is the finding of self-similar solutions of the above given equations with the specific conditions. The main tool is the reduction of considered FPDEs to the integral equations using specific transformation.

In literature, we refer some works devoted to the considered fractional derivatives, for example, hyper-Bessel operator was used to generalize the standard process of relaxation [17] and to model fractional diffusion equations governing the law of the fractional Brownian motion [18]. Also, FPDEs with hyper-Bessel operator were considered in [19] for studying direct and inverse source problems and in [20] for non-local problem of mixed type equation. Furthermore, there are different works related to applications of ErdélyiKober and Hilfer fractional derivatives such as fractional diffusion with Erdélyi-Kober derivative [21] and higher order partial differential equations with Hilfer fractional derivatives [22], for more details see the reference therein.

The rest of the paper is organized as follows. In the next section, we recall preliminaries related to some fractional derivatives. The main results are given in Section 3. The conclusion of the work is given in the last section.

\section{Preliminaries}

In this section, we present some basic definitions on fractional operators and their properties that are used further in this article.
Definition 1 ( [1]). The Riemann-Liouville fractional integral of order $\alpha>0$ is defined by

$$
I_{a t}^{\alpha} f(t)=\frac{1}{\Gamma(\alpha)} \int_{a}^{t}(t-s)^{\alpha-1} f(s) d s, \quad \alpha>0 .
$$

Definition 2 ( [23]). The right-sided Hilfer fractional derivative of order $\alpha$ and type $\delta$ is defined as

$$
D_{0 t}^{\alpha, \delta} f(t)=I_{0 t}^{\delta(n-\alpha)} \frac{d^{n}}{d t^{n}} I_{0 t}^{(1-\delta)(n-\alpha)} f(t),
$$

where $n-1<\alpha \leq n, 0 \leq \delta \leq 1$.

For $\delta=0$, Hilfer fractional derivative is reduced to the Riemann-Liouville fractional derivative, i.e; $D_{0 t}^{\alpha, \delta} f(t)=D_{0 t}^{\alpha} f(t)$.

Now, we recall the following property [24]

$$
\begin{aligned}
& I_{a^{+}}^{\sigma} D_{a^{+}}^{\sigma} f(t)=I_{a^{+}}^{\alpha} D_{a^{+}}^{\alpha, \delta} f(t) \\
& =f(t)-\sum_{k=0}^{n-1} \frac{(t-a)^{\sigma-k-1}}{\Gamma(\sigma-k)} D_{a^{+}}^{n-k-1} I_{a^{+}}^{n-\sigma} f(a),
\end{aligned}
$$

where $\sigma=\alpha+\delta-\alpha \delta$.

Definition 3. ( [25]) The left and right-sided Erdélyi-Kober fractional integrals of order $\alpha$, respectively, are defined as follows:

$$
\begin{aligned}
& I_{\beta}^{\gamma, \alpha} f(t)= \\
& \frac{\beta}{\Gamma(\alpha)} t^{-\beta(\gamma+\alpha)} \int_{0}^{t}\left(t^{\beta}-s^{\beta}\right)^{\alpha-1} s^{\beta(\gamma+1)-1} f(s) d s, \\
& J_{\beta}^{\gamma, \alpha} f(t)= \\
& \frac{\beta}{\Gamma(\alpha)} t^{\beta \gamma} \int_{t}^{\infty}\left(s^{\beta}-t^{\beta}\right)^{\alpha-1} s^{-\beta(\gamma+\alpha-1)-1} f(s) d s,
\end{aligned}
$$

where $\alpha, \beta>0$ and $\gamma \in \mathbb{R}$.

Definition 4. ( [25]) The left and right-sided Erdélyi-Kober fractional derivatives of order $\alpha$, respectively, are given by $(n-1<\alpha<n, n \in \mathbb{N})$

$$
D_{\beta}^{\gamma, \alpha} f(t)=\prod_{j=1}^{n}\left(\gamma+j+\frac{1}{\beta} t \frac{d}{d t}\right) I_{\beta}^{\gamma+\alpha, n-\alpha} f(t),
$$

and

$$
P_{\beta}^{\gamma, \alpha} f(t)=\prod_{j=0}^{n-1}\left(\gamma+j-\frac{1}{\beta} t \frac{d}{d t}\right) J_{\beta}^{\gamma+\alpha, n-\alpha} f(t),
$$

where $\gamma \in \mathbb{R}, \beta>0$. 
The following property of Erdélyi-Kober fractional operators [25]

$$
\begin{gathered}
I_{\beta}^{\gamma, \alpha} x^{\lambda \beta} f(x)=x^{\lambda \beta} I_{\beta}^{\gamma+\lambda, \alpha} f(x), \\
I_{\beta}^{\gamma, \alpha} D_{\beta}^{\gamma, \alpha} f(x)=f(x)-\sum_{k=0}^{n-1} c_{k} x^{-\beta(1+\gamma+k)},
\end{gathered}
$$

are true, where

$$
\begin{aligned}
c_{k}= & \frac{\Gamma(n-k)}{\Gamma(\alpha-k)} \lim _{x \rightarrow 0} x^{\beta(1+\gamma+k)} \times \\
& \prod_{i=k+1}^{n-1}\left(1+\gamma+i+\frac{1}{\beta} x \frac{d}{d x}\right) I_{\beta}^{\gamma+\alpha, n-\alpha} f(x) .
\end{aligned}
$$

Furthermore, the Erdélyi-Kober fractional operators of power function are needed in the computations [26]:

$$
\begin{gathered}
P_{\beta}^{\tau, \alpha} t^{p}=\frac{\Gamma(\alpha+\tau-p / \beta)}{\Gamma(\tau-p / \beta)} t^{p}, \tau-p / \beta \\
J_{\beta}^{\tau, \alpha} t^{p}=\frac{\Gamma(\tau-p / \beta)}{\Gamma(\alpha+\tau-p / \beta)} t^{p}, \tau-p / \beta>0 \\
I_{\beta}^{\gamma, \alpha} t^{p}=\frac{\Gamma(\gamma+1+p / \beta)}{\Gamma(\alpha+\gamma+1+p / \beta)} t^{p}, \gamma+1+p / \beta>0 .
\end{gathered}
$$

Definition 5. ( [27]) The hyper-Bessel operator of order order $0<\alpha<1$, is defined as

$$
\left\{\begin{array}{l}
\left.t^{\theta} \frac{d}{d t}\right)^{\alpha} f(t)= \\
(1-\theta)^{\alpha} t^{-(1-\theta) \alpha} I_{1-\theta}^{0,-\alpha} f(t), \text { if } \theta<1, \\
(\theta-1)^{\alpha} I_{1-\theta}^{-1,-\alpha} t^{(1-\theta) \alpha} f(t), \text { if } \theta>1 .
\end{array}\right.
$$

Note that $I_{\beta}^{0,-\alpha}:=D_{\beta}^{-\alpha, \alpha}$ and when $\theta=0$, this operator coincides with the Riemann-Liouville fractional derivative.

Also, we need to recall the generalized Wright function:

Definition 6. ( [9, 28]) The generalized Wright function is defined by the series expansion:

$$
W_{(\mu, a),(\nu, b)}:=\sum_{k=0}^{\infty} \frac{z^{k}}{\Gamma(a+\mu k) \Gamma(b+\nu k)},
$$

where

$\nu, \mu \in \mathbb{R}, a, b \in \mathbb{C}$.

\section{Main Result}

\subsection{Fractional differential equation involving Hilfer derivative}

Consider a time and space-fractional PDE

$$
D_{0 t}^{\alpha, \delta} u(t, x)=D_{0 x}^{\beta, \delta} u(t, x), 0<\alpha \leq 1,1<\beta \leq 2,
$$

with the following conditions:

$$
\begin{aligned}
& \frac{\partial}{\partial x} I_{0 x}^{2-m} u\left(t, 0^{+}\right)=a t^{\gamma+\alpha(1-m) / \beta}, \\
& I_{0 x}^{2-m} u\left(t, 0^{+}\right)=b t^{\gamma+\alpha(2-m) / \beta},
\end{aligned}
$$

where $a, b$ are constants and $m=\beta+\delta-\beta \delta$.

We start by using similarity method to FPDE (13) to determine a symmetry group of scaling transformations. We introduce new independent and dependent variables

$$
\bar{t}=\lambda^{b} t, \quad \bar{x}=\lambda x, \quad \bar{u}=\lambda^{c} u .
$$

The time fractional derivative becomes $\left(\sigma_{1}=\right.$ $\left.\alpha+\delta-\alpha \delta, \delta_{1}=\delta(1-\alpha)\right)$

$$
\begin{aligned}
& D_{t}^{\alpha, \delta} \bar{u}(\bar{t}, \bar{x})=I_{t}^{\delta_{1}} D_{t}^{\sigma_{1}} \bar{u}(\bar{t}, \bar{x}) \\
& =I_{t}^{\delta_{1}}\left(\frac{1}{\Gamma\left(1-\sigma_{1}\right)} \frac{\partial}{\partial t} \int_{0}^{t}(t-s)^{-\sigma_{1}}\right) \bar{u}\left(\lambda^{b} s, \bar{x}\right) d s \\
& =I_{t}^{\delta_{1}}\left(\frac{\lambda^{c+b}}{\Gamma\left(1-\sigma_{1}\right)} \frac{\partial}{\partial \bar{t}} \int_{0}^{\bar{t} / \lambda^{b}}\left(\lambda^{-b} \bar{t}-s\right)^{-\sigma_{1}}\right) \\
& =I_{t}^{\delta_{1}}\left(\frac{\lambda^{c+b \sigma_{1}}}{\Gamma\left(1-\sigma_{1}\right)} \frac{\partial}{\partial \bar{t}} \int_{0}^{\bar{t}}(\bar{t}-\tau)^{-\sigma_{1}}\right) \bar{u}(\tau, \bar{x}) d \tau \\
& =\frac{\lambda^{c+b \sigma_{1}}}{\Gamma\left(\delta_{1}\right)} \int_{0}^{t}(t-s)^{\delta_{1}-1} D_{\bar{t}}^{\sigma_{1}} \bar{u}\left(\lambda^{b} s, \bar{x}\right) d s \\
& =\frac{\lambda^{c+b \sigma_{1}}}{\Gamma\left(\delta_{1}\right)} \int_{0}^{\bar{t} / \lambda^{b}}\left(\bar{t} \lambda^{-b}-s\right)^{\delta_{1}-1} D_{\bar{t}}^{\sigma_{1}} \bar{u}\left(\lambda^{b} s, \bar{x}\right) d s \\
& =\frac{\lambda^{c+b \sigma_{1}-b-b\left(\delta_{1}-1\right)}}{\Gamma\left(\delta_{1}\right)} \int_{0}^{\bar{t}}(\bar{t}-\tau)^{\delta_{1}-1} D_{\bar{t}}^{\sigma_{1}} \bar{u}(\tau, \bar{x}) d \tau \\
& =\lambda^{c+b \alpha} I_{\bar{t}}^{\delta_{1}} D_{\bar{t}}^{\sigma_{1}} \bar{u}(\bar{t}, \bar{x}) \\
& =\lambda^{c+b \alpha} D_{\bar{t}}^{\alpha, \delta} \bar{u}(\bar{t}, \bar{x}) .
\end{aligned}
$$

One can do the same for the space-fractional derivative, we have

$$
D_{x}^{\beta, \delta} \bar{u}(\bar{t}, \bar{x})=\lambda^{c+\beta} D_{\bar{x}}^{\beta, \delta} \bar{u}(\bar{t}, \bar{x}) .
$$

From the above we get

$$
\begin{aligned}
& D_{t}^{\alpha, \delta} \bar{u}(\bar{t}, \bar{x})-D_{x}^{\beta, \delta} \bar{u}(\bar{t}, \bar{x}) \\
& =\lambda^{c+b \alpha} D_{\bar{t}}^{\alpha, \delta} \bar{u}(\bar{t}, \bar{x})-\lambda^{c+\beta} D_{\bar{x}}^{\beta, \delta} \bar{u}(\bar{t}, \bar{x})=0,
\end{aligned}
$$

if $b=\frac{\beta}{\alpha}$. Thus, we choose the following invariant of scaling transformation 


$$
u(t, x)=t^{\gamma} U(\eta), \quad \eta=x t^{-\alpha / \beta}, \gamma>0
$$

Now, using the above transformation, we have the following result:

Theorem 1. The transformation

$$
u(t, x)=t^{\gamma} U(\eta), \eta=x t^{-\alpha / \beta}
$$

reduces FPDE (13) to the following ODE

$$
J_{\beta / \alpha}^{\gamma+\delta_{2}, \delta_{1}} P_{\beta / \alpha}^{\gamma+\delta_{2}, \sigma_{1}} U(\eta)=D_{0 \eta}^{\beta, \delta} U(\eta)
$$

with

$$
D I_{0 \eta}^{2-m} U\left(0^{+}\right)=a \text { and } I_{0 \eta}^{2-m} U\left(0^{+}\right)=b,
$$

where $\sigma_{1}=\alpha+\delta-\alpha \delta, \delta_{2}=1-\sigma_{1}$ and $\delta_{1}=$ $\delta(1-\alpha)$.

Proof. We begin by calculating the timefractional derivative in terms of $U(\eta)$ using transformation (15). Using the definition of Hilfer fractional derivative (11) for $n=1$, we have

$$
D_{0 t}^{\alpha, \delta} u(t, x)=I^{\delta_{1}} \frac{\partial}{\partial t} I^{\delta_{2}} t^{\gamma} U\left(x t^{-\alpha / \beta}\right) .
$$

Now, using the substitution $\tau=t\left(\frac{\eta}{s}\right)^{\beta / \alpha}$, the second integral of (18) can be reduced as follows:

$$
\begin{aligned}
& I^{\delta_{2}} t^{\gamma} U\left(x t^{-\alpha / \beta}\right) \\
& =\frac{1}{\Gamma\left(\delta_{2}\right)} \int_{0}^{t}(t-\tau)^{\delta_{2}-1} \tau^{\gamma} U\left(x \tau^{-\alpha / \beta}\right) d \tau \\
& =\frac{\beta t^{\delta_{2}+\gamma} \eta^{\beta / \alpha(\gamma+1)}}{\alpha \Gamma\left(\delta_{2}\right)} \int_{\eta}^{\infty}\left(s^{\beta / \alpha}-\eta^{\beta / \alpha}\right)^{\delta_{2}-1} \times \\
& \quad s^{-\beta / \alpha\left(\gamma+\delta_{2}\right)-1} U(s) d s \\
& =t^{\delta_{2}+\gamma} J_{\beta / \alpha}^{\gamma+1, \delta_{2}} U(\eta) .
\end{aligned}
$$

Then, taking the derivative of the above integral, we arrive to the following

$$
\begin{aligned}
& \frac{d}{d t} I^{\delta_{2}} t^{\gamma} U\left(x t^{-\alpha / \beta}\right) \\
& =t^{\delta_{2}+\gamma-1}\left(\gamma+\delta_{2}-\frac{\alpha}{\beta} \eta \frac{d}{d \eta}\right) J_{\beta / \alpha}^{\gamma+1, \delta_{2}} U(\eta) \\
& =t^{\delta_{2}+\gamma-1} P_{\beta / \alpha}^{\gamma+\delta_{2}, \sigma_{1}} U(\eta) .
\end{aligned}
$$

Using the above result and proceeding the same as above using substitution $\tau=t\left(\frac{\eta}{s}\right)^{\beta / \alpha}$ and relation $z=x \tau^{-\alpha / \beta}$, the expression in (18) becomes

$$
\begin{aligned}
& I^{\delta_{1}} \frac{d}{d t} I^{\delta_{2}} t^{\gamma} U\left(x t^{-\alpha / \beta}\right) \\
& =\frac{1}{\Gamma\left(\delta_{1}\right)} \int_{0}^{t}(t-\tau)^{\delta_{1}-1} \tau^{\delta_{2}+\gamma-1} P_{\beta / \alpha}^{\gamma+\delta_{2}, \sigma_{1}} U(z) d \tau \\
& =\frac{\beta t^{\gamma+\delta_{2}+\delta_{1}-1} \eta^{\beta / \alpha\left(\gamma+\delta_{2}\right)}}{\alpha \Gamma\left(\delta_{1}\right)} \int_{\eta}^{\infty}\left(s^{\beta / \alpha}-\eta^{\beta / \alpha}\right)^{\delta_{1}-1} \\
& \times s^{-\beta / \alpha\left(\gamma+\delta_{2}+\delta_{1}-1\right)-1} P_{\beta / \alpha}^{\gamma+\delta_{2}, \sigma_{1}} U(s) d s .
\end{aligned}
$$

The power $\gamma+\delta_{2}+\delta_{1}-1=\gamma-\alpha$ and hence the time fractional derivative can be written as

$$
D_{0 t}^{\alpha, \delta} u(t, x)=t^{\gamma-\alpha} J_{\beta / \alpha}^{\gamma+\delta_{2}, \delta_{1}} P_{\beta / \alpha}^{\gamma+\delta_{2}, \sigma_{1}} U(\eta) .
$$

Next, we compute the space-fractional derivative in terms of $U(\eta)$

$$
D_{0 x}^{\beta, \delta} u(t, x)=t^{\gamma} I^{\delta_{4}} \frac{\partial^{2}}{\partial x^{2}} I^{\delta_{3}} U\left(x t^{-\alpha / \beta}\right),
$$

where $\delta_{3}=(2-\beta)(1-\delta)$ and $\delta_{4}=\delta(2-\beta)$. We use the substitution $\xi=s t^{-\alpha / \beta}$, then the inner integral of (19) can be written as

$$
\begin{aligned}
& I^{\delta_{3}} U\left(x t^{-\alpha / \beta}\right)=\frac{1}{\Gamma\left(\delta_{3}\right)} \int_{0}^{x}(x-s)^{\delta_{3}-1} U\left(s t^{-\alpha / \beta}\right) d s \\
& =\frac{t^{\alpha \delta_{3} / \beta}}{\Gamma\left(\delta_{3}\right)} \int_{0}^{\eta}(\eta-\xi)^{\delta_{3}-1} U(\xi) d \xi=t^{\alpha \delta_{3} / \beta} I^{\delta_{3}} U(\eta) .
\end{aligned}
$$

Computing second derivative of the above gives

$$
\frac{\partial^{2}}{\partial x^{2}} I^{(2-\beta)(1-\delta)} U\left(x t^{-\alpha / \beta}\right)=t^{\alpha\left(\delta_{3}-2\right) / \beta} \frac{d^{2}}{d \eta^{2}} I^{\delta_{3}} U(\eta),
$$

since

$\frac{d U}{d x}=\frac{d U}{d \eta} \frac{d \eta}{d x}$

Now, we do the same for the first integral of (19) with $\xi=s t^{-\alpha / \beta}$ and $z=s t^{-\alpha / \beta}$, we obtain

$$
\begin{aligned}
& I^{\delta_{4}} \frac{\partial^{2}}{\partial x^{2}} I^{\delta_{3}} U\left(x t^{-\alpha / \beta}\right) \\
& =\frac{t^{\alpha\left(\delta_{3}-2\right) / \beta}}{\Gamma\left(\delta_{4}\right)} \int_{0}^{x}(x-s)^{\delta_{4}-1} \frac{d^{2}}{d z^{2}} I^{\delta_{3}} U(z) d s \\
& =\frac{t^{\alpha\left(\delta_{3}+\delta_{4}-2\right) / \beta}}{\Gamma\left(\delta_{4}\right)} \int_{0}^{\eta}(\eta-\xi)^{\delta_{4}-1} \frac{d^{2}}{d \xi^{2}} I^{\delta_{3}} U(\xi) d \xi \\
& =t^{-\alpha} I^{\delta_{4}} \frac{d^{2}}{d \eta^{2}} I^{\delta_{3}} U(\eta) .
\end{aligned}
$$

Thus, space-fractional derivative can be written as

$$
D_{0 x}^{\beta, \delta} u(t, x)=t^{\gamma-\alpha} D_{0 \eta}^{\beta, \delta} U(\eta)
$$


Substituting the time and space-fractional derivatives after transformation, we get the desired ordinary differential equation (16).

The solution of the fractional ordinary differential equation (16) is given in the next theorem.

Theorem 2. The solution of FPDE (13) using transformation (15) with conditions (17) has the following form

$$
\begin{aligned}
u(t, x) & =t^{\gamma}\left[a \eta^{m-1} \Gamma(\gamma+1-\alpha(m-1) / \beta) \times\right. \\
& W_{(\beta, m),(-\alpha, \gamma+1-\alpha(m-1) / \beta)}\left(\eta^{\beta}\right) \\
& \left.+b \eta^{m-2} \Gamma(\gamma+1-\alpha(m-2) / \beta)\right) \times \\
& \left.W_{(\beta, m-1),(-\alpha, \gamma+1-\alpha(m-2) / \beta)}\left(\eta^{\beta}\right)\right],
\end{aligned}
$$

where $m=\beta+\delta-\delta \beta, \eta=x t^{-\alpha / \beta}$,

$$
\sum_{k=0}^{\infty} \frac{W_{(\beta, m),(-\alpha, \gamma+1-\alpha(m-2) / \beta)}\left(\eta^{\beta}\right)=}{\Gamma(m+k \beta) \Gamma(\gamma+1-k \alpha-(m-1) \alpha / \beta)}
$$

and

$$
\sum_{k=0}^{\infty} \frac{\eta_{(\beta, m-1),(-\alpha, \gamma+1-\alpha(m-2) / \beta)}\left(\eta^{\beta}\right)=}{\Gamma(m-1+k \beta) \Gamma(\gamma+1-k \alpha-(m-2) \alpha / \beta)} .
$$

Proof. Applying Riemann-Liouville fractional integral $I^{\beta}$ to both sides of differential equation (16) and using property (2), we have

$U(\eta)=\frac{a \eta^{m-1}}{\Gamma(m)}+\frac{b \eta^{m-2}}{\Gamma(m-1)}+I^{\beta} J_{\beta / \alpha}^{\gamma+\delta_{2}, \delta_{1}} P_{\beta / \alpha}^{\gamma+\delta_{2}, \sigma_{1}} U(\eta)$

Then, the solution can obtained using successive iterations method. We set

$$
U^{0}(\eta)=\frac{a \eta^{m-1}}{\Gamma(m)}+\frac{b \eta^{m-2}}{\Gamma(m-1)},
$$

so the nth term $U^{n}$ can be written as

$$
U^{n}(\eta)=U^{0}(\eta)+I^{\beta} J_{\beta / \alpha}^{\gamma+\delta_{2}, \delta_{1}} P_{\beta / \alpha}^{\gamma+\delta_{2}, \sigma_{1}} U^{n-1}(\eta)
$$

Now, we compute $U^{1}$ as follows:

$$
U^{1}(\eta)=U^{0}(\eta)+I^{\beta} J_{\beta / \alpha}^{\gamma+\delta_{2}, \delta_{1}} P_{\beta / \alpha}^{\gamma+\delta_{2}, \sigma_{1}} U^{0}(\eta)
$$

Using properties (9) and (10), we calculate the following

$$
\begin{aligned}
& I^{\beta} J_{\beta / \alpha}^{\gamma+\delta_{2}, \delta_{1}} P_{\beta / \alpha}^{\gamma+\delta_{2}, \sigma_{1}} U^{0}(\eta)= \\
& I^{\beta} J_{\beta / \alpha}^{\gamma+\delta_{2}, \delta_{1}}\left[\frac{a \eta^{m-1}}{\Gamma(m)} \frac{\Gamma\left(\gamma+\delta_{2}+\sigma_{1}-(m-1) \alpha / \beta\right)}{\Gamma\left(\gamma+\delta_{2}-(m-1) \alpha / \beta\right)}\right. \\
& \left.+\frac{b \eta^{m-2}}{\Gamma(m-1)} \frac{\Gamma\left(\gamma+\delta_{2}+\sigma_{1}-(m-2) \alpha / \beta\right)}{\Gamma\left(\gamma+\delta_{2}-(m-2) \alpha / \beta\right)}\right] \\
& =I^{\beta}\left[\frac{a \eta^{m-1}}{\Gamma(m)} \frac{\Gamma\left(\gamma+\delta_{2}+\sigma_{1}-(m-1) \alpha / \beta\right)}{\Gamma\left(\gamma+\delta_{2}+\delta_{1}-(m-1) \alpha / \beta\right)}\right. \\
& \left.+\frac{b \eta^{m-2}}{\Gamma(m-1)} \frac{\Gamma\left(\gamma+\delta_{2}+\sigma_{1}-(m-2) \alpha / \beta\right)}{\Gamma\left(\gamma+\delta_{2}+\delta_{1}-(m-2) \alpha / \beta\right)}\right] \\
& =\left[\frac{a \eta^{m+\beta-1}}{\Gamma(m+\beta)} \frac{\Gamma(\gamma+1-(m-1) \alpha / \beta)}{\Gamma(\gamma+1-\alpha-(m-1) \alpha / \beta)}+\right. \\
& \left.\frac{b \eta^{m+\beta-2}}{\Gamma(m+\beta-1)} \frac{\Gamma(\gamma+1-(m-2) \alpha / \beta)}{\Gamma(\gamma+1-\alpha-(m-2) \alpha / \beta)}\right] .
\end{aligned}
$$

Hence, $U^{1}(\eta)$ is given by

$$
\begin{aligned}
& U^{1}(\eta)=a\left(\frac{1}{\Gamma(m)} \eta^{m-1}+\eta^{m+\beta-1}\right. \\
& \left.\frac{\Gamma(\gamma+1-(m-1) \alpha / \beta)}{\Gamma(m+\beta) \Gamma(\gamma+1-\alpha-(m-1) \alpha / \beta)}\right) \\
& +b\left(\frac{1}{\Gamma(m-1)} \eta^{m-2}+\eta^{m+\beta-2}\right. \\
& \left.\frac{\Gamma(\gamma+1-(m-2) \alpha / \beta)}{\Gamma(m+\beta-1) \Gamma(\gamma+1-\alpha-(m-2) \alpha / \beta)}\right) .
\end{aligned}
$$

Similarly, we compute $U^{2}(\eta)$

$$
\begin{aligned}
& U^{2}(\eta)=U^{0}(\eta)+I^{\beta} J_{\beta / \alpha}^{\gamma+\delta_{2}, \delta_{1}} P_{\beta / \alpha}^{\gamma+\delta_{2}, \sigma_{1}} U^{1}(\eta) \\
& =U^{0}(\eta)+I^{\beta} J_{\beta / \alpha}^{\gamma+\delta_{2}, \delta_{1}} P_{\beta / \alpha}^{\gamma+\delta_{2}, \sigma_{1}} \times \\
& {\left[U^{0}(\eta)+\frac{a \Gamma(\gamma+1-(m-1) \alpha / \beta) \eta^{m+\beta-1}}{\Gamma(m+\beta) \Gamma(\gamma+1-\alpha-(m-1) \alpha / \beta)}\right.} \\
& \left.+\frac{b \Gamma(\gamma+1-(m-2) \alpha / \beta) \eta^{m+\beta-2}}{\Gamma(m+\beta-1) \Gamma(\gamma+1-\alpha-(m-2) \alpha / \beta)}\right] .
\end{aligned}
$$

One can check that

$$
\begin{gathered}
I^{\beta} J_{\beta / \alpha}^{\gamma+\delta_{2}, \delta_{1}} P_{\beta / \alpha}^{\gamma+\delta_{2}, \sigma_{1}} \eta^{m+\beta-1}=\eta^{m+2 \beta-1} \times \\
\Gamma(m+\beta) \Gamma(\gamma+1-\alpha-(m-1) \alpha / \beta) \\
\Gamma(m+2 \beta) \Gamma((\gamma+1-2 \alpha-(m-1) \alpha / \beta)
\end{gathered}
$$

and

$$
\begin{aligned}
& I^{\beta} J_{\beta / \alpha}^{\gamma+\delta_{2}, \delta_{1}} P_{\beta / \alpha}^{\gamma+\delta_{2}, \sigma_{1}} \eta^{m+\beta-2}=\eta^{m+2 \beta-2} \times \\
& \frac{\Gamma(m+\beta-1) \Gamma(\gamma+1-\alpha-(m-2) \alpha / \beta)}{\Gamma(m+2 \beta-1) \Gamma(\gamma+1-2 \alpha-(m-2) \alpha / \beta)} .
\end{aligned}
$$

Thus, 


$$
\begin{aligned}
& U^{2}(\eta)=a\left(\frac{1}{\Gamma(m)} \eta^{m-1}+\eta^{m+\beta-1} \times\right. \\
& \frac{\Gamma(\gamma+1-(m-1) \alpha / \beta)}{\Gamma(m+\beta) \Gamma(\gamma+1-\alpha-(m-1) \alpha / \beta)} \\
& \left.+\frac{\Gamma(\gamma+1-(m-1) \alpha / \beta) \eta^{m+2 \beta-1}}{\Gamma(m+2 \beta) \Gamma(\gamma+1-2 \alpha-(m-1) \alpha / \beta)}\right) \\
& +b\left(\frac{1}{\Gamma(m-1)} \eta^{m-2}+\eta^{m+\beta-2} \times\right. \\
& \frac{\Gamma(\gamma+1-(m-2) \alpha / \beta) \eta^{m+\beta-2}}{\Gamma(m+\beta-1) \Gamma(\gamma+1-\alpha-(m-2) \alpha / \beta)} \\
& \left.+\frac{\Gamma(\gamma+1-(m-2) \alpha / \beta) \eta^{m+2 \beta-2}}{\Gamma(m+2 \beta-1) \Gamma(\gamma+1-2 \alpha-(m-2) \alpha / \beta)}\right) .
\end{aligned}
$$

We similarly compute $U^{3}(\eta)$ and get

$$
\begin{aligned}
& U^{3}(\eta)=a\left(\frac{1}{\Gamma(m)} \eta^{m-1}+\right. \\
& \frac{\Gamma(\gamma+1-(m-1) \alpha / \beta) \eta^{m+\beta-1}}{\Gamma(m+\beta) \Gamma((\gamma+1-\alpha-(m-1) \alpha / \beta)} \\
& +\frac{\Gamma(\gamma+1-(m-1) \alpha / \beta) \eta^{m+2 \beta-1}}{\Gamma(m+2 \beta) \Gamma(\gamma+1-2 \alpha-(m-1) \alpha / \beta)} \\
& \left.+\frac{\Gamma(\gamma+1-(m-1) \alpha / \beta) \eta^{m+3 \beta-1}}{\Gamma(m+3 \beta) \Gamma(\gamma+1-3 \alpha-(m-1) \alpha / \beta)}\right)+ \\
& +b\left(\frac{1}{\Gamma(m-1)} \eta^{m-2}+\right. \\
& \frac{\Gamma(\gamma+1-(m-2) \alpha / \beta) \eta^{m+\beta-2}}{\Gamma(m+\beta-1) \Gamma(\gamma+1-\alpha-(m-2) \alpha / \beta)} \\
& +\frac{\Gamma(\gamma+1-(m-2) \alpha / \beta) \eta^{m+2 \beta-2}}{\Gamma(m+2 \beta-1) \Gamma(\gamma+1-2 \alpha-(m-2) \alpha / \beta)} \\
& \left.+\frac{\Gamma(\gamma+1-(m-2) \alpha / \beta) \eta^{m+3 \beta-2}}{\Gamma(m+3 \beta-1) \Gamma(\gamma+1-3 \alpha-(m-2) \alpha / \beta)}\right) .
\end{aligned}
$$

Now, we can write the $n$th term as follows:

$$
\begin{aligned}
& \sum_{k=0}^{U^{n}}(\eta)=a \eta^{m-1} \Gamma(\gamma+1-(m-1) \alpha / \beta) \times \\
& \sum_{k=0}^{n} \\
& \sum_{k=0}^{n} \frac{\eta^{\beta k}}{\Gamma(m+k \beta) \Gamma(\gamma+1-k \alpha-(m-1) \alpha / \beta))} \\
& \Gamma(\gamma+1-(m-2) \alpha / \beta) \times \\
& \eta^{\beta k}
\end{aligned}
$$

As $n$ goes to infinity, then

$$
\begin{aligned}
U(\eta) & =a \eta^{m-1} \Gamma(\gamma+1-(m-1) \alpha / \beta) \\
& \times W_{(\beta, m),(-\alpha, \gamma+1-(m-1) \alpha / \beta)}\left(\eta^{\beta}\right) \\
& +b \eta^{m-2} \Gamma(\gamma+1-(m-2) \alpha / \beta) \\
& \times W_{(\beta, m-1),(-\alpha, \gamma+1-(m-2) \alpha / \beta)}\left(\eta^{\beta}\right) .
\end{aligned}
$$

Substituting $U(\eta)$ in the transformation (15), we get the desired solution (20).

Remark 1. For $\delta=0$, Hilfer fractional derivative is reduced to Riemann-Liouville fractional derivative and this case was considered by Luchko and Gorenflo in [9]. The ordinary differential equation becomes

$$
J_{\beta / \alpha}^{\gamma, 1-\alpha} P_{\beta / \alpha}^{\gamma, \alpha} U(\eta)=D_{x}^{\beta} U(\eta)
$$

and one can check that the solution has the following form

$$
\begin{aligned}
U(\eta) & =a \eta^{\beta-1} \Gamma(\gamma+1-\alpha+\alpha / \beta) \\
& \times W_{(\beta, \beta),(-\alpha, \gamma+1-\alpha+\alpha / \beta)}\left(\eta^{\beta}\right) \\
& +b \eta^{\beta-2} \Gamma(\gamma+1-\alpha+2 \alpha / \beta) \\
& \times W_{(\beta, \beta-1),(-\alpha, \gamma+1-\alpha+2 \alpha / \beta)}\left(\eta^{\beta}\right),
\end{aligned}
$$

which coincides with their result.

For $\beta=2$ and $0<\alpha \leq 2$, this case was studied by Buckwar and Luchko, for more details see [6, 28].

Remark 2. One may consider the same problem with $n-1<\alpha \leq n$ and $n-1<\beta \leq n$ :

$$
D_{0 t}^{\alpha, \delta} u(t, x)=D_{0 x}^{\beta, \delta} u(t, x)
$$

with conditions

$$
D^{n-k-1} I_{0 x}^{n-m} u\left(t, 0^{+}\right)=c_{n}, \quad m=\beta+\delta-\beta \delta,
$$

and then use the same transformation in (15) to find the exact solution. Proceeding the same, the solution has the following form:

$$
\begin{aligned}
& u(t, x)=t^{\gamma} \sum_{i=0}^{n} c_{i} \eta^{m-i-1} \times \\
& \Gamma(\gamma+1-(m-i-1) \alpha / \beta) \\
& \times W_{(\beta, m-i),(-\alpha, \gamma+1-(m-i-1) \alpha / \beta)}\left(\eta^{\beta}\right) .
\end{aligned}
$$

\subsection{Fractional differential equation involving hyper-Bessel operator}

Consider the problem

$$
\left(t^{\theta} \frac{\partial}{\partial t}\right)^{\alpha} u(t, x)=x^{-\beta \rho} \frac{\partial^{\beta}}{\partial x^{\beta}} u(t, x)
$$

with the boundary conditions:

$$
\begin{aligned}
& \lim _{x \rightarrow 0} x^{\rho(\beta-1)}\left(1-\beta+\frac{1}{\rho} x \frac{d}{d x}\right) I_{\rho}^{0,2-\beta} u(t, x)= \\
& U_{0} t^{\gamma-\alpha \rho(\beta-1) / \beta}
\end{aligned}
$$

$$
\begin{aligned}
& \lim _{x \rightarrow 0} x^{\rho(\beta-2)}\left(2-\beta+\frac{1}{\rho} x \frac{d}{d x}\right) I_{\rho}^{0,2-\beta} u(t, x)= \\
& U_{1} t^{\gamma-\alpha \rho(\beta-2) / \beta}
\end{aligned}
$$


where $1<\beta \leq 2,0<\alpha \leq 1, \rho=1-\theta$, $\left(t^{\theta} \frac{\partial}{\partial t}\right)^{\alpha}$ stands for hyper-Bessel operator defined by (12) and $\frac{\partial^{\beta}}{\partial x^{\beta}}=D_{\rho}^{-\beta, \beta}$ represents the left-sided Erdélyi-Kober fractional derivative.

First, we use similarity method for FPDE 21 to determine a symmetry group of scaling transformations. We introduce new independent and dependent variables as before

$$
\bar{t}=\lambda^{b} t, \quad \bar{x}=\lambda x, \quad \bar{u}=\lambda^{c} u .
$$

The time fractional derivative becomes

$$
\begin{aligned}
& \left(t^{\theta} \frac{\partial}{\partial t}\right)^{\alpha} \bar{u}(\bar{t}, \bar{x}) \\
& =\rho^{\alpha} t^{-\rho \alpha} I^{0,-\alpha} \rho \bar{u}(\bar{t}, \bar{x}) \\
& =\rho^{\alpha} t^{-\rho \alpha} D_{\rho, t}^{-\alpha, \alpha} \bar{u}(\bar{t}, \bar{x}) \\
& =\rho^{\alpha} t^{-\rho \alpha}\left(1-\alpha+\frac{1}{\rho} t \frac{d}{d t}\right) I_{\rho, t}^{0,1-\alpha} \bar{u}(\bar{t}, \bar{x}) \\
& =\rho^{\alpha} t^{-\rho \alpha}\left(1-\alpha+\frac{1}{\rho} t \frac{d}{d t}\right) \frac{\rho t^{-\rho(1-\alpha)}}{\Gamma(1-\alpha)} \int_{0}^{t}(t-s)^{-\alpha} \\
& \times s^{\rho-1} \bar{u}\left(s \lambda^{b}, \bar{x}\right) d s \\
& =\rho^{\alpha} \lambda^{b \rho \alpha} \bar{t}^{-\rho \alpha}\left(1-\alpha+\frac{1}{\rho} \bar{t} \frac{d}{d \bar{t}}\right) \frac{\rho \lambda^{b \rho(1-\alpha)} \bar{t}^{-\rho(1-\alpha)}}{\Gamma(1-\alpha)} \\
& \times \int_{0}^{\bar{t} / \lambda^{b}}\left(\bar{t} \lambda^{-b}-s\right)^{-\alpha} s^{\rho-1} \bar{u}\left(s \lambda^{b}, \bar{x}\right) d s \\
& =\rho^{\alpha} \lambda^{b \rho \alpha} \bar{t}^{-\rho \alpha}\left(1-\alpha+\frac{1}{\rho} \bar{t} \frac{d}{d \bar{t}}\right) \frac{\rho \lambda^{b \rho \alpha} t^{-\rho(1-\alpha)}}{\Gamma(1-\alpha)} \\
& \times \int_{0}^{\bar{t}}(\bar{t}-\tau)^{-\alpha} \tau^{\rho-1} \bar{u}(\tau, \bar{x}) d \tau \\
& =\rho^{\alpha} \lambda^{b \rho \alpha} \bar{t}^{-\rho \alpha}\left(1-\alpha+\frac{1}{\rho} \bar{t} \frac{d}{d \bar{t}}\right) I_{\rho, \bar{t}}^{0,1-\alpha} \bar{u}(\bar{t}, \bar{x}) \\
& =\lambda^{b \rho \alpha}\left(\bar{t}^{\theta} \frac{\partial}{\partial \bar{t}}\right)^{\alpha} \bar{u}(\bar{t}, \bar{x}) .
\end{aligned}
$$

Similarly, we do for the space-fractional derivative and deduce

$$
\begin{aligned}
& D_{\rho, x}^{-\beta, \beta} \bar{u}(\bar{t}, \bar{x})= \\
& \prod_{k=0}^{1}\left(1-\beta+k+\frac{1}{\rho} x \frac{d}{d x}\right) I_{\rho, x}^{0,2-\beta} \bar{u}(\bar{t}, \bar{x}) \\
& =\prod_{k=0}^{1}\left(1-\beta+k+\frac{1}{\rho} \bar{x} \frac{d}{d \bar{x}}\right) \frac{\rho \lambda^{\rho(2-\beta)} \bar{x}^{-\rho(2-\beta)}}{\Gamma(2-\beta)} \\
& \times \int_{0}^{\bar{x} / \lambda}(\bar{x} / \lambda-s)^{1-\beta} s^{\rho-1} \bar{u}(\bar{t}, s \lambda) d s
\end{aligned}
$$

$$
\begin{aligned}
& =\prod_{k=0}^{1}\left(1-\beta+k+\frac{1}{\rho} \bar{x} \frac{d}{d \bar{x}}\right) \frac{\rho \bar{x}^{-\rho(2-\beta)}}{\Gamma(2-\beta)} \\
& \times \int_{0}^{\bar{x}}(\bar{x}-z)^{1-\beta} z^{\rho-1} \bar{u}(\bar{t}, z) d z \\
& =\prod_{k=0}^{1}\left(1-\beta+k+\frac{1}{\rho} \bar{x} \frac{d}{d \bar{x}}\right) I_{\rho, \bar{x}}^{0,2-\beta} \bar{u}(\bar{t}, \bar{x}) \\
& =D_{\rho, \bar{x}}^{-\beta, \beta} \bar{u}(\bar{t}, \bar{x}) .
\end{aligned}
$$

From the above we get

$$
\begin{aligned}
& \left(t^{\theta} \frac{\partial}{\partial t}\right)^{\alpha} \bar{u}(\bar{t}, \bar{x})-x^{\rho \beta} D_{\rho, x}^{-\beta, \beta} \bar{u}(\bar{t}, \bar{x}) \\
& =\lambda^{b \rho \alpha}\left(\bar{t}^{\theta} \frac{\partial}{\partial \bar{t}}\right)^{\alpha} \bar{u}(\bar{t}, \bar{x})-\lambda^{\rho \beta} \bar{x}^{\rho \beta} D_{\rho, \bar{x}}^{-\beta, \beta} \bar{u}(\bar{t}, \bar{x}) \\
& =0
\end{aligned}
$$

if $b=\frac{\beta}{\alpha}$. Thus, we choose the following invariant of scaling transformation

$$
u(t, x)=t^{\gamma} U(\eta), \quad \eta=x t^{-\alpha / \beta} .
$$

The result related to equation (21) is given in the following theorem;

Theorem 3. The transformation given by (15) reduces the FPDE (21) to the following ODE

$$
\rho^{\alpha} \eta^{\beta \rho} P_{\rho \beta / \alpha}^{1-\alpha+\gamma / \rho, \alpha} U(\eta)=D_{\rho}^{-\beta, \beta} U(\eta),
$$

with

$$
\lim _{\eta \rightarrow 0} \eta^{\rho(\beta-1)}\left(1-\beta+\frac{1}{\rho} \eta \frac{d}{d \eta}\right) I_{\rho}^{0,2-\beta} U(\eta)=U_{0}
$$

and

$$
\lim _{\eta \rightarrow 0} \eta^{\rho(\beta-2)}\left(2-\beta+\frac{1}{\rho} \eta \frac{d}{d \eta}\right) I_{\rho}^{0,2-\beta} U(\eta)=U_{1} .
$$

Proof. We start by rewriting the time-hyperBessel operator using definition (12):

$$
\left(t^{\theta} \frac{\partial}{\partial t}\right)^{\alpha} u(t, x)=\rho^{\alpha} t^{-\rho \alpha} I_{\rho}^{0,-\alpha} t^{\gamma} U(\eta) .
$$

Then, make change of variable $\tau=t\left(\frac{\eta}{s}\right)^{\beta / \alpha}$ and simplify as follows 


$$
\begin{aligned}
& \rho^{\alpha} t^{-\rho \alpha} D_{\rho}^{-\alpha, \alpha} t^{\gamma} U(\eta) \\
& =\rho^{\alpha} t^{-\rho \alpha}\left(1-\alpha+\frac{1}{\rho} t \frac{d}{d t}\right) I_{\rho}^{0,1-\alpha} t^{\gamma} U\left(x t^{-\alpha / \beta}\right) \\
& =\rho^{\alpha} t^{-\rho \alpha}\left(1-\alpha+\frac{1}{\rho} t \frac{d}{d t}\right) \frac{\rho t^{-\rho(1-\alpha)}}{\Gamma(1-\alpha)} \\
& \times \int_{0}^{t}\left(t^{\rho}-\tau^{\rho}\right)^{-\alpha} \tau^{\rho-1+\gamma} U\left(x \tau^{-\alpha / \beta}\right) d \tau \\
& =\rho^{\alpha} t^{-\rho \alpha}\left(1-\alpha+\frac{1}{\rho} t \frac{d}{d t}\right) \frac{\rho \beta t^{\gamma} \eta^{\rho \beta / \alpha(1+\gamma / \rho)}}{\alpha \Gamma(1-\alpha)} \\
& \times \int_{\eta}^{\infty}\left(s^{\rho \beta / \alpha}-\eta^{\rho \beta / \alpha}\right)^{-\alpha} s^{-\rho \beta(1-\alpha+\gamma / \rho)-1} U(s) d s \\
& =\rho^{\alpha} t^{\gamma-\rho \alpha}\left(1-\alpha+\frac{1}{\rho} t \frac{d}{d t}\right) J_{\rho \beta / \alpha}^{1+\gamma / \rho, 1-\alpha} U(\eta) .
\end{aligned}
$$

Thus, we arrive

$$
\begin{aligned}
& \rho^{\alpha} t^{-\rho \alpha} D_{\rho}^{-\alpha, \alpha} t^{\gamma} U(\eta) \\
& =\rho^{\alpha} t^{\gamma-\rho \alpha}\left(1-\alpha+\frac{\gamma}{\rho}-\frac{\alpha}{\rho \beta} \eta \frac{d}{d \eta}\right) J_{\rho \beta / \alpha}^{1+\gamma / \rho, 1-\alpha} U(\eta) \\
& =\rho^{\alpha} t^{\gamma-\rho \alpha} P_{\rho \beta / \alpha}^{1-\alpha+\gamma / \alpha, \alpha} U(\eta),
\end{aligned}
$$

where $J_{\rho \beta / \alpha}^{1+\gamma / \rho, 1-\alpha}$ and $P_{\rho \beta / \alpha}^{1-\alpha+\gamma / \alpha, \alpha}$ are right-sided Erdélyi-Kober fractional operators. Similarly, we transform the space fractional derivative

$$
\begin{aligned}
& D_{\rho}^{-\beta, \beta} u(t, x) \\
& =\prod_{k=0}^{1}\left(1-\beta+k+\frac{1}{\rho} x \frac{d}{d x}\right) I_{\rho}^{0,2-\beta} t^{\gamma} U\left(x t^{-\alpha / \beta}\right),
\end{aligned}
$$

and substitute $s=x\left(\frac{z}{\eta}\right)$ in the above in the integral as follows:

$$
\begin{aligned}
& I_{\rho}^{0,2-\beta} t^{\gamma} U\left(x t^{-\alpha / \beta}\right) \\
& =\frac{\rho t^{\gamma} x^{-\rho(2-\beta)}}{\Gamma(2-\beta)} \int_{0}^{x}\left(x^{\rho}-s^{\rho}\right)^{1-\beta} s^{\rho-1} U\left(s t^{-\alpha / \beta}\right) d s \\
& =\frac{\rho t^{\gamma} \eta^{-\rho(2-\beta)}}{\Gamma(2-\beta)} \int_{0}^{\eta}\left(\eta^{\rho}-z^{\rho}\right)^{1-\beta} z^{\rho-1} U(z) d z \\
& =t^{\gamma} I_{\rho}^{0,2-\beta} U(\eta) .
\end{aligned}
$$

Hence,

$$
\begin{aligned}
& D_{\rho}^{-\beta, \beta} u(t, x) \\
& =t^{\gamma} \prod_{k=0}^{1}\left(1-\beta+k+\frac{1}{\rho} \eta \frac{d}{d \eta}\right) I_{\rho}^{0,2-\beta} U(\eta) \\
& =t^{\gamma} D_{\rho}^{-\beta, \beta} U(\eta)
\end{aligned}
$$

Finally, substituting the transformed time and space fractional derivatives in differential equation (21), we get

$$
\rho^{\alpha} t^{\gamma-\rho \alpha} P_{\rho \beta / \alpha}^{1-\alpha+\gamma / \alpha, \alpha} U(\eta)=x^{-\rho \beta} t^{\gamma} D_{\rho}^{-\beta, \beta} U(\eta)
$$

which can be written as ordinary differential equation (24).

In the next theorem, we give the self-similar solution (invariant solution) of equation (21):

Theorem 4. The solution of FPDE (21) using transformation (15) with conditions (25)-(26) has the following form

$$
\begin{aligned}
u(t, x) & =t^{\gamma}\left[\eta^{\rho(\beta-1)} \Gamma\left(\frac{\gamma}{\rho}+1+\frac{\alpha}{\beta}-\alpha\right)\right. \\
& \times W_{(\beta, \beta),\left(-\alpha, \frac{\gamma}{\rho}+1+\frac{\alpha}{\beta}-\alpha\right)}\left(\rho^{\alpha} \eta^{\beta}\right) \\
& \left.+\eta^{\rho(\beta-2)} \Gamma\left(\frac{\gamma}{\rho}+1+\frac{2 \alpha}{\beta}-\alpha\right)\right) \\
& \left.\times W_{(\beta-1, \beta),\left(-\alpha, \frac{\gamma}{\rho}+1+\frac{2 \alpha}{\beta}-\alpha\right)}\left(\rho^{\alpha} \eta^{\beta}\right)\right]
\end{aligned}
$$

Proof. Applying Erdélyi-Kober fractional integral to both sides of equation (24) and using the property (8), we have

$$
\begin{aligned}
U(\eta) & =c_{0} \eta^{\rho(\beta-1)}+c_{1} \eta^{\rho(\beta-2)} \\
& +\rho^{\alpha} I_{\rho}^{-\beta, \beta} \eta^{\beta \rho} P_{\rho \beta / \alpha}^{1-\alpha+\gamma / \rho, \alpha} U(\eta),
\end{aligned}
$$

where $c_{0}=\frac{U_{0}}{\Gamma(\beta)}$ and $c_{1}=\frac{U_{1}}{\Gamma(\beta-1)}$.

Also, using property (7), we get

$$
\begin{aligned}
U(\eta) & =c_{0} \eta^{\rho(\beta-1)}+c_{1} \eta^{\rho(\beta-2)} \\
& +\rho^{\alpha} \eta^{\beta \rho} I_{\rho}^{0, \beta} P_{\rho \beta / \alpha}^{1-\alpha+\gamma / \rho, \alpha} U(\eta) .
\end{aligned}
$$

Note that $I_{\rho}^{-\beta, \beta} \eta^{\beta \rho}=\eta^{\beta \rho} I_{\rho}^{0, \beta}$, see [25].

To find the solution of the above equation, we use successive iteration method. We start with

$$
U^{0}=c_{0} \eta^{\rho(\beta-1)}+c_{1} \eta^{\rho(\beta-2)}
$$

and

$$
U^{n}(\eta)=U^{0}+\rho^{\alpha} \eta^{\beta \rho} I_{\rho}^{0, \beta} P_{\rho \beta / \alpha}^{1-\alpha+\gamma / \rho, \alpha} U^{n-1}(\eta) .
$$

The first iteration is

$$
\begin{gathered}
U^{1}(\eta)=U^{0}+\rho^{\alpha} \eta^{\beta \rho} I_{\rho}^{0, \beta} P_{\rho \beta / \alpha}^{1-\alpha+\gamma / \rho, \alpha} U^{0}(\eta) \\
=U^{0}+\rho^{\alpha} \eta^{\beta \rho} I_{\rho}^{0, \beta} P_{\rho \beta / \alpha}^{1-\alpha+\gamma / \rho, \alpha} \\
\quad\left(c_{0} \eta^{\rho(\beta-1)}+c_{1} \eta^{\rho(\beta-2)}\right)
\end{gathered}
$$

Compute the second term of $U^{1}(\eta)$ using property (11), we have 


$$
\begin{aligned}
& I_{\rho}^{0, \beta} P_{\rho \beta / \alpha}^{1-\alpha+\gamma / \rho, \alpha} \eta^{\rho(\beta-1)} \\
& =I_{\rho}^{0, \beta} \eta^{\rho(\beta-1)} \frac{\Gamma\left(\frac{\gamma}{\rho}+1+\frac{\alpha}{\beta}-\alpha\right)}{\Gamma\left(\frac{\gamma}{\rho}+1+\frac{\alpha}{\beta}-2 \alpha\right)} \\
& =\eta^{\rho(\beta-1)} \frac{\Gamma(\beta) \Gamma\left(\frac{\gamma}{\rho}+1+\frac{\alpha}{\beta}-\alpha\right)}{\Gamma(2 \beta) \Gamma\left(\frac{\gamma}{\rho}+1+\frac{\alpha}{\beta}-2 \alpha\right)},
\end{aligned}
$$

and similarly we do for the last term

$$
\begin{aligned}
& I_{\rho}^{0, \beta} P_{\rho \beta / \alpha}^{1-\alpha+\gamma / \rho, \alpha} \eta^{\rho(\beta-2)} \\
& =\eta^{\rho(\beta-2)} \frac{\Gamma(\beta-1) \Gamma\left(\frac{\gamma}{\rho}+1+\frac{2 \alpha}{\beta}-\alpha\right)}{\Gamma(2 \beta-1) \Gamma\left(\frac{\gamma}{\rho}+1+\frac{2 \alpha}{\beta}-2 \alpha\right)} .
\end{aligned}
$$

Substituting back the above results in $U^{1}(\eta)$, we get

$$
\begin{aligned}
U^{1}(\eta) & =c_{0}\left[\eta^{\rho(\beta-1)}+\rho^{\alpha} \eta^{\rho(2 \beta-1)}\right. \\
& \left.\times \frac{\Gamma(\beta) \Gamma\left(\frac{\gamma}{\rho}+1+\frac{\alpha}{\beta}-\alpha\right)}{\Gamma(2 \beta) \Gamma\left(\frac{\gamma}{\rho}+1+\frac{\alpha}{\beta}-2 \alpha\right)}\right] \\
& +c_{1}\left[\eta^{\rho(\beta-2)}+\rho^{\alpha} \eta^{\rho(2 \beta-2)}\right. \\
& \left.\times \frac{\Gamma(\beta-1) \Gamma\left(\frac{\gamma}{\rho}+1+\frac{2 \alpha}{\beta}-\alpha\right)}{\Gamma(2 \beta-1) \Gamma\left(\frac{\gamma}{\rho}+1+\frac{2 \alpha}{\beta}-2 \alpha\right)}\right] .
\end{aligned}
$$

Repeating the same procedure, one can compute $U^{2}(\eta)$ :

$$
U^{2}(\eta)=U^{0}+\rho^{\alpha} \eta^{\beta \rho} I_{\rho}^{0, \beta} P_{\rho \beta / \alpha}^{1-\alpha+\gamma / \rho, \alpha} U^{1}(\eta)
$$

So,

$$
\begin{aligned}
& I_{\rho}^{0, \beta} P_{\rho \beta / \alpha}^{1-\alpha+\rho / \rho, \alpha} \eta^{\rho(2 \beta-1)} \\
& =I_{\rho}^{0, \beta} \eta^{\rho(2 \beta-1)} \frac{\Gamma\left(\frac{\gamma}{\rho}+1+\frac{\alpha}{\beta}-2 \alpha\right)}{\Gamma\left(\frac{\gamma}{\rho}+1+\frac{\alpha}{\beta}-3 \alpha\right)} \\
& =\eta^{\rho(2 \beta-1)} \frac{\Gamma(2 \beta) \Gamma\left(\frac{\gamma}{\rho}+1+\frac{\alpha}{\beta}-2 \alpha\right)}{\Gamma(3 \beta) \Gamma\left(\frac{\gamma}{\rho}+1+\frac{\alpha}{\beta}-3 \alpha\right)}
\end{aligned}
$$

and

$$
\begin{aligned}
& I_{\rho}^{0, \beta} P_{\rho \beta / \alpha}^{1-\alpha+\gamma / \rho, \alpha} \eta^{\rho(2 \beta-2)} \\
& =\eta^{\rho(2 \beta-2)} \frac{\Gamma(2 \beta-1) \Gamma\left(\frac{\gamma}{\rho}+1+\frac{2 \alpha}{\beta}-2 \alpha\right)}{\Gamma(3 \beta-1) \Gamma\left(\frac{\gamma}{\rho}+1+\frac{2 \alpha}{\beta}-3 \alpha\right)} .
\end{aligned}
$$

Thus, $U^{2}(\eta)$ can be written as follows

$$
\begin{aligned}
& U^{2}(\eta) \\
& =c_{0}\left[\eta^{\rho(\beta-1)}+\rho^{\alpha} \eta^{\rho(2 \beta-1)} \frac{\Gamma(\beta) \Gamma\left(\frac{\gamma}{\rho}+1+\frac{\alpha}{\beta}-\alpha\right)}{\Gamma(2 \beta) \Gamma\left(\frac{\gamma}{\rho}+1+\frac{\alpha}{\beta}-2 \alpha\right)}\right. \\
& \left.+\rho^{2 \alpha} \eta^{\rho(3 \beta-1)} \frac{\Gamma(\beta) \Gamma\left(\frac{\gamma}{\rho}+1+\frac{\alpha}{\beta}-\alpha\right)}{\Gamma(3 \beta) \Gamma\left(\frac{\gamma}{\rho}+1+\frac{\alpha}{\beta}-3 \alpha\right)}\right] \\
& +c_{1}\left[\eta^{\rho(\beta-2)}\right. \\
& +\rho^{\alpha} \eta^{\rho(2 \beta-2)} \frac{\Gamma(\beta-1) \Gamma\left(\frac{\gamma}{\rho}+1+\frac{2 \alpha}{\beta}-\alpha\right)}{\Gamma(2 \beta-1) \Gamma\left(\frac{\gamma}{\rho}+1+\frac{2 \alpha}{\beta}-2 \alpha\right)} \\
& \left.+\rho^{2 \alpha} \eta^{\rho(3 \beta-2)} \frac{\Gamma(\beta-1) \Gamma\left(\frac{\gamma}{\rho}+1+\frac{2 \alpha}{\beta}-\alpha\right)}{\Gamma(3 \beta-1) \Gamma\left(\frac{\gamma}{\rho}+1+\frac{2 \alpha}{\beta}-3 \alpha\right)}\right] .
\end{aligned}
$$

Now, the $n$th iteration can be written as

$$
\begin{aligned}
& U^{n}(\eta)=U_{0} \Gamma\left(\frac{\gamma}{\rho}+1+\frac{\alpha}{\beta}-\alpha\right) \\
& \sum_{k=0}^{n} \frac{\rho^{k \alpha} \eta^{\rho(k \beta-1)}}{\Gamma(k+1) \beta) \Gamma\left(\frac{\gamma}{\rho}+1+\frac{\alpha}{\beta}-(k+1) \alpha\right)} \\
& +U_{1} \Gamma\left(\frac{\gamma}{\rho}+1+\frac{2 \alpha}{\beta}-\alpha\right) \\
& \sum_{k=0}^{n} \frac{\rho^{k \alpha} \eta^{\rho(k \beta-2)}}{\Gamma(k+1) \beta-1) \Gamma\left(\frac{\gamma}{\rho}+1+\frac{2 \alpha}{\beta}-(k+1) \alpha\right)}
\end{aligned}
$$

and as $n$ approaches infinity, we have

$$
\begin{aligned}
U(\eta) & =U_{0} \Gamma\left(\frac{\gamma}{\rho}+1+\frac{\alpha}{\beta}-\alpha\right) \eta^{\rho(\beta-1)} \\
& W_{(\beta, \beta),\left(-\alpha, \frac{\gamma}{\rho}+1+\frac{\alpha}{\beta}-\alpha\right)}\left(\rho^{\alpha} \eta^{\beta}\right) \\
& +U_{1} \Gamma\left(\frac{\gamma}{\rho}+1+\frac{2 \alpha}{\beta}-\alpha\right) \eta^{\rho(\beta-2)} \\
& W_{(\beta-1, \beta),\left(-\alpha, \frac{\gamma}{\rho}+1+\frac{2 \alpha}{\beta}-\alpha\right)}\left(\rho^{\alpha} \eta^{\beta}\right) .
\end{aligned}
$$

Substituting $U(\eta)$ in the transformation (15), we obtain the desired solution (27).

Remark 3. Particular case when $\rho=1$, the fractional derivatives in (21) are reduced to RiemannLiouville fractional derivatives and the problem was considered by Luchko and Gorenflo as mentioned above, for more details see the reference therein.

\section{Conclusion}

To summarize, Fractional Partial Differential Equations (FPDEs) involving hyper-Bessel, Erdélyi-Kober and Hilfer fractional derivatives were main targets in this investigation. Using special transformation (see (15)) we first reduced the considered FPDEs to the fractional ODEs (see Theorem 1 and 3) and then we solved these ODEs using successive iterative method (see Theorem 2 
and 4). The obtained self-similar solutions are expressed in terms of generalized Wright type function.

Our motivation is based on possible usage of subdiffusion equations with such special fractional operators by specialists in applied mathematics who may deal with such sub-diffusion equations. Moreover, we believe that suggested approach can be applied for investigation of more general FPDEs and also in studying the symmetry group analysis of these of derivatives.

\section{References}

[1] Kilbas A.A., Srivastava H.M. \& Trujillo J.J. (2006). Theory and Applications of fractional differential equations, Amsterdam: Elsevier.

[2] Singh J. (2020). Analysis of fractional blood alcohol model with composite fractional derivative. Chaos, Solitons and Fractals, 140, 110127.

[3] Singh J., Kumar D. \& Baleanu D. (2020). A new analysis of fractional fish farm model associated with Mittag-Leffler-type kernel. International Journal of Biomathematics, 13(2), 2050010.

[4] Karimov, E.T. (2020). Frankl-Type Problem for a Mixed Type Equation with the Caputo Fractional Derivative. Lobachevskii Journal of Mathematics 41, 1829-1836.

[5] Uchaikin, V.V. Fractional Derivatives for Physicists and Engineers, Volume I Background and Theory Volume II Applications, Springer, 2013.

[6] Buckwar, E., Luchko, Y. (1998). Invariance of a partial differential equation of fractional order under the Lie group of scaling transformations. Journal of Mathematical Analysis and Applications, 227(1), 81-97.

[7] Stanyukovich, K.P. Unsteady Motion of Continuous Media, Oxford: Pergamon Press, 1959.

[8] Barenblatt, G. I.Scaling, self-similarity, and intermediate asymptotics: dimensional analysis and intermediate asymptotics (No. 14). Cambridge University Press, 1996.

[9] Luchko, Y. \& Gorenflo, R. (1998). Scaleinvariant solutions of a partial differential equation of fractional order. Fractional Calculus and Applied Analysis, 1(1), 63-78.

[10] Basti B. \& Benhamidouche N. (2020). Existence results of self-similar solutions to the Caputo-types space-fractional heat equation. Surveys in Mathematics and its Applications, 15, 153-168.
[11] Gazizov, R.K., Kasatkin, A.A. \& Lukashchuk, S. Y. (2007). Continuous transformation groups of fractional differential equations. Vest. USATU, 9, 125-135.

[12] Gazizov, R. K., Kasatkin, A. A. \& Lukashchuk, S. Y. (2009). Symmetry properties of fractional diffusion equations. Physica Scripta, 2009(136), 014016.

[13] Duan, J. S., Guo, A. P. \& Yun, W. Z. (2014). Similarity solution for fractional diffusion equation. Abstract and Applied Analysis, Article ID 548126, 1-5.

[14] Silver Costa, F., Marao, J.A.P.F., AlvesSoares, J.C. \& Capelas de Oliveira, E. (2015). Similarity solution to fractional nonlinear space-time diffusion-wave equation. Journal of Mathematical Physics, 3 (2015), 033507.

[15] Bakkyaraj T. \& Sahadevan R. (2015). Group formalism of Lie transformations to timefractional partial differential equations, Pramana. Journal of Physics, 85(5), 845-860.

[16] Ruzhansky M. \& Hasanov A. (2020). SelfSimilar Solutions of Some Model Degenerate Partial Differential Equations of the Second, Third, and Fourth Order, Lobachevskii Journal of mathematics, 41, 1103-1114.

[17] Garra, R., Giusti, A., Mainardi, F., \& Pagnini, G. (2014). Fractional relaxation with time-varying coefficient. Fractional Calculus and Applied Analysis, 17(2), 424-439.

[18] Garra, R., Orsingher, E., \& Polito, F. (2015). Fractional diffusions with time-varying coefficients. Journal of Mathematical Physics, 56(9), 093301.

[19] Al-Musalhi F., Al-Salti N. \& Karimov E.T. (2018). Initial boundary value problems for a fractional differential equation with hyperBessel operator, Fractional Calculus and Applied Analysis 21(1), 200-219.

[20] Karimov, E. T. \& Toshtemirov, B. H. (2019). Tricomi type problem with integral conjugation condition for a mixed type equation with the hyper-bessel fractional differential operator. Bulletin of the Institute of Mathematics, (4), 9-14.

[21] Pagnini, G. (2012). Erdélyi-Kober fractional diffusion. Fractional calculus and applied analysis, 15(1), 117-127.

[22] Yuldashev T.K. \& Kadirkulov B.J. (2020). Nonlocal problem for a mixed type fourthorder differential equation with Hilfer fractional operator. Ural mathematical journal, 6(1), 153-167.

[23] Hilfer, R., Luchko, Y. \& Tomovski, Z. (2009). Operational method for the solution 
of fractional differential equations with generalized Riemann-Liouville fractional derivatives. Fractional Calculus and Applied Analysis, 12(3), 299-318.

[24] Furati, K. M. \& Kassim, M. D. (2012). Existence and uniqueness for a problem involving Hilfer fractional derivative. Computers and Mathematics with Applications, 64(6), 16161626.

[25] Luchko, Y. \& Trujillo, J. (2007). Caputo-type modification of the Erdélyi-Kober fractional derivative. Fractional Calculus and Applied Analysis, 10(3), 249-267.

[26] Hanna, L. M. \& Luchko, Y. F. (2014). Operational calculus for the Caputo-type fractional Erdélyi-Kober derivative and its applications. Integral Transforms and Special Functions, 25(5), 359-373.

[27] Garra, R., Giusti, A., Mainardi, F. \& Pagnini, G. (2014). Fractional relaxation with time-varying coefficient. Fractional Calculus and Applied Analysis, 17(2), 424-439.
[28] Gorenflo, R., Luchko, Y., \& Mainardi, F. (2000). Wright functions as scale-invariant solutions of the diffusion-wave equation. Journal of Computational and Applied Mathematics, 118(1-2), 175-191.

Fatma Al-Musalhi (PhD) is an instructor at Center for Preparatory Studies, Sultan Qaboos University $(S Q U)$ in Oman and also a researcher. She received the master and Ph.D degree in Mathematics at SQU. Her research interests are on Fractional differential equation and mathematical modeling.

(10) https://orcid.org/0000-0003-1108-3879

Erkinjon Karimov (DSc) is an Associate Professor at the Ferghana State University and Senior Researcher at the V.I.Romanovskiy Institute of Mathematics, Uzbekistan Academy of Sciences (part-time). In 1998 he has got his Master degree from Fergana State University, then in 2006 he has got his PhD and in 2020 DSc from the V.I. Romanovskiy Institute of Mathematics.

(10)https://orcid.org/0000-0003-4443-6300

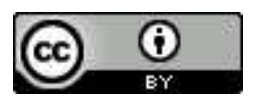

This work is licensed under a Creative Commons Attribution 4.0 International License. The authors retain ownership of the copyright for their article, but they allow anyone to download, reuse, reprint, modify, distribute, and/or copy articles in IJOCTA, so long as the original authors and source are credited. To see the complete license contents, please visit http://creativecommons.org/licenses/by/4.0/. 